\title{
The importance of being upright: Use of environmental and viewer-centered reference frames in shape discriminations of novel three-dimensional objects
}

\author{
ALINDA FRIEDMAN and DAVID LAWRENCE HALL \\ University of Alberta, Edmonton, Alberta, Canada
}

\begin{abstract}
We investigated the frame of reference that people use to make shape discriminations when their heads are either upright or tilted. Observers made same-different judgments of pairs of novel threedimensional objects that were aligned along their length within the frontal-parallel plane and rotated in depth around an axis parallel to their own axes of elongation. The aligned objects were displayed vertically, tilted $45^{\circ}$, or horizontally with respect to the environmental upright, but the distance of each pair from the upright was irrelevant to resolving the angular disparity between the stimuli for the same-different judgment. Nevertheless, when observers' heads were upright, the time to encode the stimuli was a linear function of the distance of the stimuli from the environmental upright, whereas when observers' heads were tilted $45^{\circ}$, encoding times for tilted and vertical stimuli did not differ and were faster than the times to encode horizontal stimuli. We interpreted these data to mean that observers either rotate or reference the top of an object to the environmental upright, and they can use either a gravitational or retinal reference frame to do so when either they or the objects are not upright.
\end{abstract}

Objects project different shapes on the retina virtually every time they are seen, yet people can readily make judgments about them that are based on their shape. To determine how this is done, we can examine the frame of reference used to discriminate among objects that have undergone systematic orientation transformations. One possibility is that the frame of reference is viewer centered (see, e.g., Edelman \& Bülthoff, 1992; Hinton \& Parsons, 1981; Rock, 1974; Tarr, 1995; Tarr \& Pinker, 1990), so that one of the steps involved in shape identity or discrimination judgments is to determine how an object is aligned relative to the observer's head (or retina). On this view, computational difficulties arise as a function of discrepancies between the top of the head and the top of the object. A second possibility is that observers use an environmental reference frame (see, e.g., Attneave \& Olson, 1967; Corballis, Nagourney, Shetzer, \& Stefanatos, 1978; Corballis, Zbrodoff, \& Roldan, 1976), so that difficulties arise as a function of discrepancies between the top of the object and the environmental (visual) or gravitational upright, irrespective of the

Portions of these experiments were presented at the XXV International Congress of Psychology, Brussels, in July 1992. The research reported here was supported by the Natural Sciences and Engineering Research Council of Canada. We are grateful to Marian Morry and Joy Williams for collecting the data and helping with the data analysis, and to Michael Dawson and Vince DiLollo for helpful comments on earlier versions of the manuscript. Comments by Michael Corballis, Asher Koriat, Lester Krueger, Pierre Jolicoeur, Steve Palmer, and Lynne Robertson on earlier drafts also provided helpful insights and suggestions for improvements. Requests for reprints should be addressed to A. Friedman, Department of Psychology, University of Alberta, Edmonton, AB, Canada T6G 2E9 (e-mail: alinda@psych.ualberta.ca). position of the viewer's head. The environmental upright and the gravitational upright are normally coincident, but they need not be (consider, e.g., the rod-and-frame effect; see Asch \& Witkin, 1948; DiLorenzo \& Rock, 1982).

In this paper, we present evidence in favor of yet a third alternative: When observers are judging objects on the basis of their shape, they do determine how the objects are aligned with respect to the environmental upright, perhaps because that is how the representation is ultimately organized. But the relative ease with which this relation can be determined is affected by both the orientation of the objects and the orientation of the observer.

It is easy to determine that an upright object is upright even when an observer is not, because the observer can use the gravitational vector as a cue to his/her own position as well as to the position of the stimulus (Wilson \& Jones, 1979). That is, a tilted observer can readily compute or detect that an upright object is aligned with the gravitational vector. However, when an object is not aligned with the environmental upright, it is easier to compute how the object happens to be misaligned (and then reference it to the upright) if the observer is misaligned in the same way. Again, this is because the observer can detect the relation between the top of his/her head and the environmental upright by using gravitational cues; by inference, if an observer is aligned with a tilted stimulus, the observer-environment relation is the same as the stimulus-environment relation. On this view, both the observer's and the environmental reference frames play a role in shape judgments about objects in different orientations (see, e.g., Corballis \& Roldan, 1975).

Because the environmental and observer's frames of reference coincide whenever an observer is upright, their 
independent influence can be investigated by having people tilt their heads (Attneave \& Olson, 1967; Rock, 1974; Rock \& Heimer, 1957; Rock \& Leaman, 1963). Thus, our general approach was to have observers whose heads were either upright or tilted judge the shape identity of pairs of unfamiliar three-dimensional (3-D) objects in a standard mental rotation paradigm (Cooper \& Shepard, 1973; Metzler \& Shepard, 1974; Shepard \& Metzler, 1971). We depicted the objects as having been rotated in depth, so that the head-tilt manipulation did not obviate the need to perform the mental rotation component of the task, as it does with 2-D stimuli. In Experiment 1, we established how upright observers made same-different shape identity judgments with these objects, and in Experiment 2, we determined how performance changed as a function of head tilt. Because the unfamiliar objects that we used had neither a learned top nor a prototypical orientation, we assumed that their alignment with respect to either the environment or the observer would be determined as part of the process of computing their structural description during encoding (Marr, 1982; Marr \& Nishihara, 1978). There is no need to know the identity of an object to complete this stage of processing (Tarr \& Pinker, 1990).

In the standard mental rotation paradigm, the ubiquitous finding is that judgment times and errors increase as a linear function of the angular disparity between the objects being judged (Metzler \& Shepard, 1974; Shepard \& Metzler, 1971). In process models developed for the task that assume discrete stages (Sternberg, 1969), the intercept of the function relating angular disparity to reaction time (RT) is hypothesized to reflect encoding and decision times; the slope reflects the time taken by the rotation process itself (Cooper \& Shepard, 1973; Egan, 1978; Just \& Carpenter, 1976, 1985). Most investigators have concentrated their efforts on manipulations designed to influence the slope of the rotation function (e.g., stimulus complexity; Bethell-Fox \& Shepard, 1988; Hall \& Friedman, 1994; Yuille \& Steiger, 1982). However, factors that affect the encoding of the stimulus may also be relevant to the frame of reference issue, because encoding times reflect the ease or difficulty with which the representation of the stimulus and/or its reference frame is established. We have been concerned with the orientation of the rotation axis (Friedman, Pilon, \& Gabrys, 1988; see also Parsons, 1987; Shiffrar \& Shepard, 1991) and the orientation of the stimuli relative to the environmental upright (Friedman \& Pilon, 1994), which may each plausibly affect both the slope and the intercept of the RT function.

In the earlier study (Friedman et al., 1988), the RT functions for aligned pairs of objects oriented vertically within the frontal-parallel plane (e.g., the pair in Figure 1a) had lower intercepts than did the functions for identically aligned pairs that had been tilted "away" from the frontalparallel plane via a rotation around the horizontal $(x)$ axis. We will refer to figures tilted toward or away from the frontal-parallel plane as foreshortened. In contrast to foreshortened objects, the pairs of objects in Figures $1 \mathrm{~b}$ and $\mathrm{lc}$ are rotated around the viewer's line of sight and are thus tilted within the frontal-parallel plane.

We wished to interpret the difference between intercepts produced by vertical stimuli and those produced by foreshortened stimuli as reflecting the fact that an object's orientation with respect to the vertical upright is used to set up a structural description of the object for purposes of the mental rotation task. However, it is also true that the axis structure of objects that have been tilted toward or away from the viewer may be more difficult to determine and that their parts may be more obscured than those of identical objects aligned within the picture plane (Humphrey \& Jolicoeur, 1988). More important, because the objects were aligned along their length and rotated around the axis with respect to which they were aligned, the long axis of the figures and the axis of rotation were entirely coincident for both the vertical and the foreshortened stimuli. Consequently, the obtained differences between conditions could have been due to how difficult it was to compute the two different axes, or to perform rotations around the two axes, rather than to the relative difficulty of encoding vertical as opposed to foreshortened stimuli per se with respect to the environmental upright.

To address these issues, we independently varied the angular disparity between the two stimuli in a given pair and the vertical distance between the end of one of the items in the pair and the environmental upright (Friedman \& Pilon, 1994). For example, to create a pair in which the items were $45^{\circ}$ apart but the pair as a whole was $30^{\circ}$ from upright, two vertically aligned stimuli were first both rotated $30^{\circ}$ clockwise within the picture plane; then, the rightmost stimulus in the pair was rotated a further $45^{\circ}$. In the conditions of interest for the present argument, the stimuli were all parallel to the frontal-parallel plane, and they were rotated with respect to each other, as well as with respect to the upright, around the axis coincident to the line of sight of the viewer. Thus, the axis of rotation was constant across conditions, and all the parts of the objects were equally visible at each of the distances from the upright and through all of the putative intermediate states (i.e., no parts mentally disappeared and reappeared; see Metzler \& Shepard, 1974).

Both distance from the upright and angular disparity affected the time to make the same-different judgments. Indeed, although angular disparity certainly accounted for the major portion of the variance $(78.6 \%$, averaged across all experiments), vertical distance accounted for an additional $9.4 \%$ of unique variance. In the conditions of most relevance to the present research, correct decision times were best described by Equation 1, in which the constant represents the value of the intercept when the predictor variables equal zero, and $\beta_{2 k}$ represents the change in the intercept (in milliseconds) as a function of the change in distance from the vertical (in degrees). Thus, the intercept is given by $\beta_{0}+\beta_{2}$ (vertical distance) ${ }_{k}$, whether or not the factors are additive. In addition, when the factors are additive, $\beta_{1 j}$ is the slope for the angular disparity functions for all of the distances from the vertical; otherwise, it is the 
slope for the angular disparity function when vertical distance is zero (see Friedman \& Pilon, 1994).

$$
\begin{aligned}
\mathrm{RT}= & \left.\beta_{0}+\beta_{1} \text { (angular disparity }\right)_{j} \\
& \left.+\beta_{2} \text { (vertical distance }\right)_{k}
\end{aligned}
$$

Across all experiments, the angular disparity between the objects contributed an average of $15.1 \mathrm{msec} / \mathrm{deg}$ to the slope of the decision time function, and the distance of the pair as a whole from the vertical contributed an average of $4.3 \mathrm{msec} / \mathrm{deg}$ to its intercept. These findings support three related interpretations: Encoding the stimuli in the mental rotation task involves (1) rotating or (2) referencing the objects to the environmental upright, or (3) rotating the subjective (upright) reference frame itself to match the reference frame of the rotated objects (see, e.g., Robertson, Palmer, \& Gomez, 1987). Although we could not distinguish among these possibilities, because the rotation axis was the same in all conditions, the experiments ruled out the computational difficulty of either determining or rotating around a particular axis as contributing to the effect of distance from the vertical. And because all the stimuli were aligned within the frontal-parallel plane, the experiments also ruled out the possibility that differences between conditions were caused by differences in the visibility of the parts of the objects. However, we still did not know whether stimuli that are closer to the vertical are easier to encode because they are aligned with the gravitational (environmental) upright or because they are aligned with the (upright) observers. We examined this issue in Experiment 2.

It is also the case that because the stimulus objects were rotated within the picture plane, not in depth, observers might have performed the task partially or entirely on the basis of 2-D information (see Jolicoeur, Regehr, Smith, \& Smith, 1985). That is, we could not ensure that observers encoded and used the actual 3-D structure of the stimuli to perform the task (see Pilon \& Friedman, 1995). Indeed, although issues concerning frames of reference are usually meant to pertain to 3 -D objects, in virtually all of the studies conducted to date, 2-D stimuli have been used (e.g., letters of the alphabet, Robertson et al. [1987]; line drawings of natural objects, McMullen \& Jolicoeur [1990, 1992]). It thus seemed important not only to use 3-D stim- uli, but to ensure that depth information was required for successful performance.

\section{EXPERIMENT 1}

In Experiment 1, we examined the performance of upright observers making shape identity judgments to pairs of vertical, tilted, and horizontal stimuli like those shown in Figures 1a-1c. Both members of a pair were parallel to the frontal-parallel plane and rotated in depth around their long axes. The pairs in each condition were otherwise physically identical in every respect (e.g., size, perspective, amount obscured or not, etc.) and were separated by the same physical distance. Because these stimuli must be mentally rotated in depth to achieve successful performance, it is reasonable to assume that the mental representations of the stimuli must somehow reflect their 3-D structure. Thus, the three-dimensionality of the stimuli was integral to the task.

Something important to note about the stimuli is that the angular disparity between the objects - that is, the amount to be mentally rotated - was exactly the same at each distance from the vertical upright. This was different from experiments in which letters or other familiar or unfamiliar 2-D drawings have been used to study the frame of reference issue, because the amount that 2-D stimuli are tilted away from the vertical is the angular disparity factor. In the present study, however, the distance from the vertical was independent of the angular disparity between the stimuli, so vertical distance should not affect the mental rotation component of the task. But distance from the vertical should affect the processes used to set up the representation of the stimuli if observers rotated or referenced the stimuli to either their own or to the environmental upright before engaging in mental rotation processes involved in the shape discrimination per se.

Note that because the stimuli were always aligned along their length, having to find matching ends (e.g., Just \& Carpenter, 1976) should not have been an important factor contributing to performance, just as it had not been a factor previously (Friedman \& Pilon, 1994, Experiment 3 ). Thus, we anticipated that the angular disparity and vertical distance factors would again be additive. Moreover, there was nothing about the physical arrangement of our

\section{$\begin{array}{lll}\text { VERTICAL } & \text { TILT } & \text { HORIZONTAL }\end{array}$}

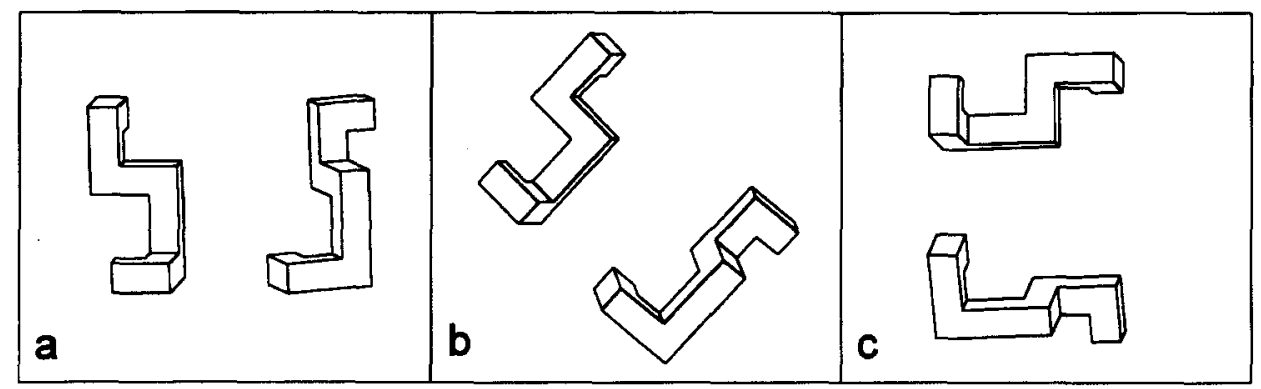

Figure 1. Examples of stimuli used in the vertical, tilted, and horizontal conditions of both experiments. 
stimuli per se that could influence the results: The two vertical, tilted, and horizontal stimuli in each pair were all the same distance from each other, and the literature on the ballistic nature of saccadic eye movements indicates no difference in speed or duration between horizontal (left-right), vertical (up-down), or oblique saccades (Taumer, Lemb, \& Namislo, 1976, p. 172; Yarbus, 1967, p. 142), although latencies of upward saccades are shorter than downward saccades, with left-right latencies in between (so that the latency differences average out; Heywood \& Churcher, 1980). It is also important to note that the different trials were pairs of mirror-image isomorphs (the plane of the mirror passed through the central arm and was parallel to the long axis of the figures). Since both Corballis et al. (1976) and McMullen and Jolicoeur (1990) found that left-right and/or mirror-image discriminations were influenced by an environmental frame of reference, we anticipated that that was what we would find too. Thus, if observers are trying to rotate or reference the ends of the objects to the upright of either the environmental or the subjective reference frame, then, when the observers themselves are upright, the farther from the environmental/ subjective upright the top of the object is, the longer it will take to encode. In this case, vertical stimuli should be encoded most quickly and accurately, followed by tilted stimuli, and tilted stimuli should be encoded more quickly than horizontal stimuli.

An alternative possibility is that observers encode objects with respect to a frame of reference that corresponds to the standard $x y z$ coordinate system (Just \& Carpenter, 1985), so that objects oriented either vertically or horizontally should be easier to encode than tilted objects, because vertical and horizontal objects are each already parallel to a major axis of the system. There are certainly enough data in the literature indicating that tilted stimuli are more difficult than either horizontal or vertical stimuli in a wide variety of tasks (e.g., Appelle, 1972; Lasaga \& Garner, 1983; Palmer \& Hemenway, 1978; Shiffrar \& Shepard, 1991; but see Corballis \& Roldan, 1975). The vertical stimuli in the present experiment might have a further advantage over horizontal stimuli because vertical stimuli also happen to be aligned with the observer's head, but tilted stimuli should be the most difficult if observers are working with a standard $x y z$ "picture plane" representation.

\section{Method}

Subjects. Six male and 6 female volunteers from the University of Alberta Department of Psychology subject pool served as observers. They received $5 \notin$ per correct trial for both practice and experimental trials, and they could earn up to $\$ 23.40$ (Canadian) for participating.

Stimuli and Design. The stimuli were constructed using a computer graphics package (MOVIE.BYU; Christiansen \& Stephenson, 1983). We created three stimulus conditions in which the pairs were physically identical in every respect except for orientation from the upright (see Figures la-1c). Only one shape (and its mirror image) was used in all conditions.

To create the stimuli for the vertical condition, the stimulus shape and its mirror image (designated $\mathrm{A}$ and $\mathrm{B}$, respectively) were first completely aligned within the frontal-parallel plane (i.e., no depth was apparent). Then, to reveal the 3-D structure, the starting posi- tion stimuli were first rotated $10^{\circ}$ around the horizontal axis and then $10^{\circ}$ around the vertical axis. Finally, for each shape, a sequence of 12 views was generated by successive rotations of the starting position stimulus $30^{\circ}$ around the vertical axis. The tilted stimuli were constructed by rotating each vertical stimulus $45^{\circ}$ in the picture plane, and the horizontal stimuli were constructed by rotating each vertical stimulus $90^{\circ}$ in the picture plane.

From each sequence of 12 views of Shapes $A$ and B, 12 same pairs were formed at each of six relative angular disparities $\left(30^{\circ}, 60^{\circ}, 90^{\circ}\right.$, $120^{\circ}, 150^{\circ}$, and $180^{\circ}$ ). Thus, there were 144 same pairs ( 2 shapes $X$ 6 relative disparities $\times 12$ pairs each) consisting of AA or BB shapes. Similarly, 144 different pairs were constructed from either $\mathrm{AB}$ or $\mathrm{BA}$ shapes, by using the same views and relative angular disparities as those for the same pairs. Finally, a given figure from each pair could be on either the left or the right, for a total of 576 stimulus pairs possible in each of the vertical, tilted, and horizontal conditions. A given observer saw 144 of the 576 possible pairs per vertical, tilted, and horizontal condition, for a total of 432 pairs. The stimuli were selected so that across 4 different observers, all 576 pairs within each distance from the vertical condition were seen exactly once. Each item in a pair was a maximum of $3.7^{\circ}$ wide and $4.8^{\circ}$ high; a pair of stimuli centered on the screen subtended a maximum visual angle of approximately $10.1^{\circ}$.

For any given observer, the 144 pairs seen in the vertical, tilted, and horizontal conditions were identical in every respect (other than their orientation from the upright). The observers saw these pairs randomly distributed over six blocks of 72 trials each. In each block, there were 24 stimuli from each of the vertical, tilted, and horizontal conditions. Half of these were $A A$ and $B B$ pairs and half were $A B$ and $B A$ pairs, so there were two same and two different pairs at each of the six angular disparities. The stimulus pairs were randomized throughout the block, with the restriction that no more than four items in a row could require the same response. A practice block of 36 trials ( 12 in each stimulus condition) was created with the same characteristics as those of an experimental block, except that only angular disparities of $30^{\circ}, 90^{\circ}$, and $150^{\circ}$ were used.

Apparatus and Procedure. An observer was seated $96 \mathrm{~cm}$ in front of a Hewlett-Packard 1304a oscilloscopic display with greenishtint, fast-decay P15 phosphor (decay to $10 \%$ intensity at $2.8 \mu \mathrm{sec}$ after display offset); the resolution was such that lines appeared straight and continuous at all angles. A plotting device developed by Finley (1985) was used to plot the figures on the oscilloscope during each session. A computer timed the responses and controlled the display devices.

Observers placed their heads in a chinrest with two laterally placed head stops, used to ensure that they were in a comfortable position but could not tilt their heads. A set of touchplates made of two wooden boards with two metal strips across them was on the table in front of the observers. Observers rested the heels of their hands on one of the metal strips and their index fingers on raised wooden strips at the front of the boards. They responded by touching their index finger to the smaller metal plate. Observers used their preferred hand for same responses and the other hand for different responses.

Instructions for the task were read to the observers, who were then shown a demonstration in which a pair of figures was displayed on the screen and one of the figures was rotated in $30^{\circ}$ steps by successive responses on the touchplates until that figure was in the same orientation as that of the other figure. This was done for a same and different pair from all three conditions. The purpose of the demon. stration was to make the concept of mental rotation explicit and to allow practice with the touchplates.

After the demonstration, the observers received the practice trials. Each trial began with a short tone, followed $.5 \mathrm{sec}$ later by a fixation cross in the center of the screen for $1 \mathrm{sec}$. Then the cross disappeared and the figures were displayed until the observer responded. If the response was correct, the figures disappeared and the next trial began. If the response was incorrect, two beeps were sounded and the figures flashed off and then on again and remained on the screen 
for 5 additional seconds so that the observer could review his/her error. The procedure for the experimental trials was the same as for the practice trials, except that no feedback was given after incorrect responses.

After the first half of the trials, the observers were told how many trials were correct and how much they had earned and were then given approximately a 5-min rest. Then the second half of the experimental trials was given. The experimenter remained seated behind the observer throughout the session.

\section{Results}

Mean RTs were computed over correct same trials; Figure 2 shows these means as a function of angular disparity and vertical distance. It is apparent that there is no interaction between the factors, and that the vertical stimuli were responded to fastest and most accurately, followed by tilted stimuli, followed by the horizontal stimuli. All the statistical analyses confirmed these observations. The error data on same trials (Figure 2) mirrored the RT data in all important respects and indicate that there was no speed-accuracy tradeoff. The RTs and errors for different trials as a function of angular disparity and distance from the vertical are given in the Appendix.

A hierarchical regression analysis on the 18 treatment means displayed in Figure 2 showed that when the $80.6 \%$ $[F(1,16)=66.37, p<.001]$ of the variance attributable to the angular disparity between the objects was partialed out,

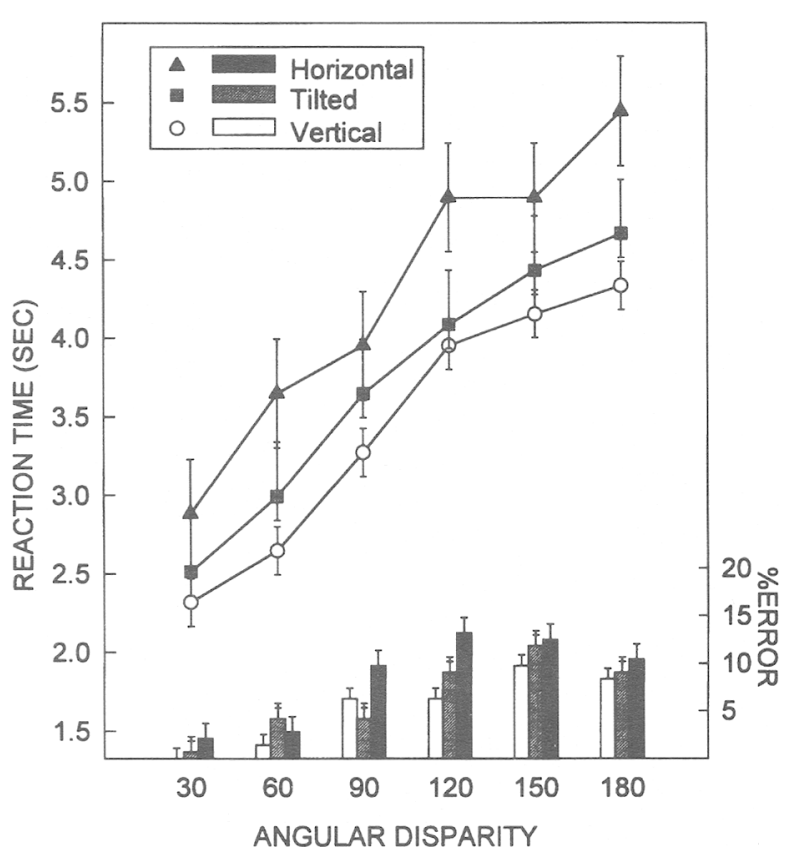

Figure 2. Reaction times and error rates on correct same trials from Experiment 1 . The error bars represent the $95 \%$ confidence intervals (Loftus \& Masson, 1994). In this figure, as well as in Figures 3 and 4, the error bars between the vertical and tilted stimuli are based on the error term for the single $d f$ contrast between those two conditions, and the error bars between the tilted and horizontal stimuli are based on the error term for that single $d f$ contrast. In this figure, as in Figure 4, the larger of the two error bars for the tilted stimuli in the percent error data is for the contrast between the tilted and the horizontal stimuli. distance from the vertical accounted for $15.3 \%$ of the additional (unique) variance $[F(1,15)=56.41, p<.001]$. The interaction term did not enter into the equation, as we had found previously (Friedman \& Pilon, 1994, Experiment 3). The resulting parameter estimates were $15.3 \mathrm{msec} /$ deg for angular disparity $\left(\beta_{1 j}\right)$ and $9.3 \mathrm{msec} / \mathrm{deg}$ for vertical distance $\left(\beta_{2 k}\right)$.

The data most relevant to issues of encoding were provided by the intercepts on both same and different trials, which were derived from regression lines computed over the angular disparity factor separately for responses to the vertical, tilted, and horizontal stimuli for each observer. The mean intercepts across observers and the results of the planned comparisons on those means are shown in Table 1 . For same trials, $98.9 \%$ of the variance in the main effect of distance from the vertical was due to the 619msec difference between the vertical and horizontal stimuli. Intercepts for the vertical stimuli were lower than those for tilted or horizontal stimuli for 9 of the $12 \mathrm{ob}$ servers, and 8 of the 12 observers had lower intercepts for the tilted stimuli than for the horizontal stimuli. For different trials, $82.1 \%$ of the variance in the main effect of distance from the vertical was also due to the difference between the vertical and horizontal stimuli. Nine of the 12 observers had lower intercepts for vertical stimuli than for tilted or horizontal stimuli, and 9 observers also had lower intercepts for tilted stimuli than for horizontal stimuli.

The analysis of the overall RT data is, of course, redundant with the regression analysis reported earlier; the mean RTs for the vertical, tilted, and horizontal stimuli and the results of the planned comparisons on those means are reported in Table 2. Neither the distance from the vertical by disparity interaction nor any of its higher order components were reliable. Indeed, the analysis of the slopes derived from the correct same RTs showed no difference across the three vertical distance conditions $[F(2,22)<1]$; the slopes for the vertical, tilted, and horizontal stimuli were 14.6, 16.7 , and $14.8 \mathrm{msec} / \mathrm{deg}$, respectively.

\section{Discussion}

Unlike some perceptual tasks in which tilted stimuli are more difficult than either horizontal or vertical stimuli (e.g., Appelle, 1972; Lasaga \& Garner, 1983; Palmer \& Hemenway, 1978), the tilted condition here produced encoding times and accuracy levels midway between those of the horizontal and vertical conditions for most of the observers (see also Corballis \& Roldan, 1975, and McMullen \& Jolicoeur, 1990). Thus, all the measures in Experiment 1, together with our previous data (Friedman \& Pilon, 1994) converge on one major conclusion: When observers are discriminating mirror-image stimuli, a constant amount of additional time is required and an increasing number of errors are made as a function of the amount that the stimulus pairs are tilted from the upright. This is so, even though distance from the upright is irrelevant to the task and the angular disparity between the stimuli continues to have its usual effect on performance. Our earlier data (Friedman \& Pilon, 1994) preclude attributing encoding time differences at different distances from the upright to the diffi- 
Table 1

Intercepts (in Milliseconds) and Planned Comparison Results for Same and Different Trials for Experiment 1

\begin{tabular}{lcc}
\hline & Same & Different \\
\hline Vertical & 1,918 & 3,934 \\
Tilted & 2,171 & 4,106 \\
Horizontal & 2,537 & 5,729 \\
Horizontal-vertical & $619^{\mathrm{a}}$ & $1,795^{\mathrm{d}}$ \\
Tilted-vertical & $253^{\mathrm{b}}$ & $972^{\mathrm{e}}$ \\
$\quad$ Horizontal-tilted & $366^{\mathrm{c}}$ & $1,623^{\mathrm{f}}$ \\
\hline${ }^{\mathrm{a}} F(1,11)=13.36, p<.01 . \quad \mathrm{b} F(1,11)=3.28, p<.10$. & ${ }^{\mathrm{c}} F(1,11)=2.17$, \\
n.s. $\mathrm{d} F(1,11)=6.07, p<.05 . \quad{ }^{\mathrm{e}} F(1,11)=0.42, \mathrm{n.s.}$ & ${ }^{\mathrm{f}} F(1,11)=6.05$, \\
$p<.05$. &
\end{tabular}

culty of determining or rotating around a particular axis, because in that study only one rotation axis was used. Further, the fact that in the present case all the rotations were depicted in depth lessens the possibility that observers used primarily 2-D information to perform the task.

\section{EXPERIMENT 2}

To examine whether the environmental or the retinal upright was the more important factor in producing the effect of distance from the vertical on intercepts, observers performed the same task as in Experiment 1 with their heads either upright or tilted. Note again that Experiment 2 is unique because even when the observer's head is aligned with the tilted stimuli, the stimuli still need to be mentally rotated to decide whether they have the same shape. In contrast, when the observer's head and a 2-D stimulus are both aligned at $45^{\circ}$ from the upright, there is basically no rotation to be done.

Even with 2-D stimuli, however, there is some confusion in the literature regarding when it is advantageous to have an observer's head aligned with a stimulus and when it is not. For example, Corballis and Roldan (1975; see also Palmer \& Hemenway, 1978; Rock, 1974) found that symmetry judgments of differently oriented dot patterns were influenced by head tilt, whereas Corballis et al. (1976) found no effect of head tilt when observers performed a mental rotation task with alphanumeric characters. Corballis et al. (1976) therefore concluded that symmetry judgments were made by using viewer-centered retinal coordinates whereas mental rotation of characters was done by using environmentally based coordinates.

McMullen and Jolicoeur (1990; see also Jolicoeur, 1985, 1988) also found evidence for task-specific reference frame effects: Performance was influenced by head tilt when observers named 2-D line drawings of familiar objects displayed at various orientations but not when they judged whether the same line drawings were facing left or right. This is an important finding, because the naming task does not require a left-right (i.e., mirror image) discrimination and so it cannot be argued that the results are due to observers' having to determine the "handedness" of the stimuli. Therefore, McMullen and Jolicoeur (1990) argued that a retinal frame is used for object identification, whereas an environmental frame is used for judgments involving left - right discriminations (see also McMullen \& Jolicoeur, 1992). Thus, on the basis of previous findings for 2-D stimuli, the most tenable expectation for the present experiment is that the head-tilt condition will be no different than the upright condition. There are two additional possibilities, however.

According to the view that the frame of reference for encoding objects more generally (e.g., 3-D objects) is viewer centered, observers always refer stimuli to themselves, so retinal coordinates should matter especially if the direction in which the stimuli are tilted does not change the fact that they need to be rotated, or the amount by which they need to be rotated. On this view, when the head is tilted in the same direction as are the tilted stimuli, that condition will be easier than both the horizontal and vertical conditions. Moreover, because the horizontal and vertical stimuli are equally far from being aligned with the observer's head, they should be equally difficult.

A third possibility is that observers use both frames of reference. That is, observers may always refer (or rotate) 3-D stimuli to the environmental vertical but the computations necessary to do this take place with respect to the orientation of the head when the head is not vertical. This possibility is reasonable, because even when a viewer is not upright, the gravitational vector remains constant relative to the environment and may be used as a frame of reference to help the viewer locate him/herself with respect to other objects in the field of view. That is, because the vestibular system allows the viewer to detect the gravitational vector irrespective of his/her position in space (Wilson \& Jones, 1979), it should be easy to compute where the environmental vertical is relative to the viewer and to the stimuli.

If observers use the gravitational vector to reference either the stimuli or their subjective reference frame to the environmental upright, then, even when the head (or body) is tilted, vertical stimuli will remain easy for the same reason that they were easy in the head-upright condition: They are already aligned with the gravitational vector. In addition, when the viewer's head is tilted, the tilted stimuli may become as easy to encode as the vertical stimuli. This is because, first, it is easy to compute where the ends of the stimuli are relative to the viewer because the stimuli are aligned with the viewer's retinal upright, and sec-

Table 2

Mean Reaction Times (RT, in Milliseconds) and Planned Comparison Results for Same and Different Trials for Experiment 1

\begin{tabular}{lcc}
\hline & \multicolumn{2}{c}{ RT } \\
\cline { 2 - 3 } & Same & Different \\
\hline Vertical & 3,447 & 4,377 \\
Tilted & 3,723 & 4,633 \\
Horizontal & 4,287 & 5,441 \\
Horizontal-vertical & $840^{\mathrm{a}}$ & $1,064^{\mathrm{d}}$ \\
Tilted-vertical & $276^{\mathrm{b}}$ & $256^{\mathrm{e}}$ \\
Horizontal-tilted & $564^{\mathrm{c}}$ & $808^{\mathrm{f}}$ \\
\hline a $F(1,11)=10.57, p<.01, \quad \mathrm{~b} F(1,11)=7.88, p<.025 . \quad{ }^{\mathrm{c}} F(1,11)=$ \\
$6.42, p<.025 . \quad{ }^{\mathrm{d}} F(1,11)=6.88, p<.025$. & ${ }^{\mathrm{e}} F(1,11)=1.40$, n.s. \\
$\mathrm{f} F(1,11)=9.26, p<.025$. & &
\end{tabular}


ond, it is easy to infer the relation between the self and the upright by using vestibular cues. On this account, the horizontal stimuli should still be the most difficult to encode. We can distinguish among these three accounts with regression analyses, because the values given to each of the vertical, tilted, and horizontal stimulus pairs change as a function of the assumptions regarding which frame of reference is being used.

\section{Method}

Subjects, Stimuli, and Design. Eight male and 8 female volunteers from the University of Alberta subject pool received $5 \notin$ per correct trial. Half the males and half the females received the head-upright condition first, and the other half of each group received the head-tilt condition first. The tilted and horizontal stimuli in Experiment 1 had been tilted from the vertical in the clockwise direction; because of the way in which the head-tilt apparatus was constructed, the stimuli for the present experiment were tilted in a counterclockwise direction. Otherwise, the stimulus pairs and counterbalancing sequences were identical to those used in Experiment 1. In addition, a given observer saw the identical stimulus sequences with his/her head in each position, though a different random order of stimuli was used for each observer

Apparatus. A head-tilt apparatus was built that consisted of a chinrest and a head support designed to allow the observer to tilt his/her head to the left. A Velcro strap was attached to the top of the head support and held the observer's head gently in place. A mechanism attached to the head support was set at a $49.5^{\circ}$ tilt (to control for the $4.5^{\circ}$ counterrolling of the eyes toward the environmental vertical due to the vestibular-ocular reflex; Corballis et al., 1976). In the head-upright condition, the observer's head was also held in position with the chinrest, headrest, and Velcro strap. The height of the chair was adjusted for each observer until he/she was comfortable in both the upright and tilted positions.

Observers were seated $101.5 \mathrm{~cm}$ away from the oscilloscope screen. This meant that each item in a stimulus pair subtended a maximum visual angle of $3.5^{\circ}$ and a maximum height of $4.5^{\circ}$; a pair of stimuli centered on the screen subtended a maximum visual angle of about $9.6^{\circ}$

\section{Results}

Preliminary analyses of the data from the same trials showed that observers became faster and less error-prone with practice, so that whichever head-tilt condition was first was slower and more errorful than whichever headtilt condition was second $[F(1,14)=5.58, p<.05$ for RT; and $F(1,14)=12.88, p<.01$ for errors]. However, presentation order did not interact with any other factor, and subsequent analyses were collapsed over this factor.

Once again, only data from correct trials were used to compute the mean RTs and the regression lines; slopes and intercepts were derived from the regression lines, and analyses of the error data mirrored those of the RT data and will not be reported. Figures 3 and 4 show the RT and error data from same trials in the head-upright and headtilted conditions, respectively, and Figure 5 shows the intercepts from same trials. Essentially, it can be seen that the head-upright condition replicated the first experiment and the head-tilted condition did not. RTs and errors for different trials are given in the Appendix.

The mean intercepts for same and different trials and the results of the planned comparisons on those means are shown in Table 3. For same trials, when observers' heads were upright, intercepts were lower for vertical stimuli than

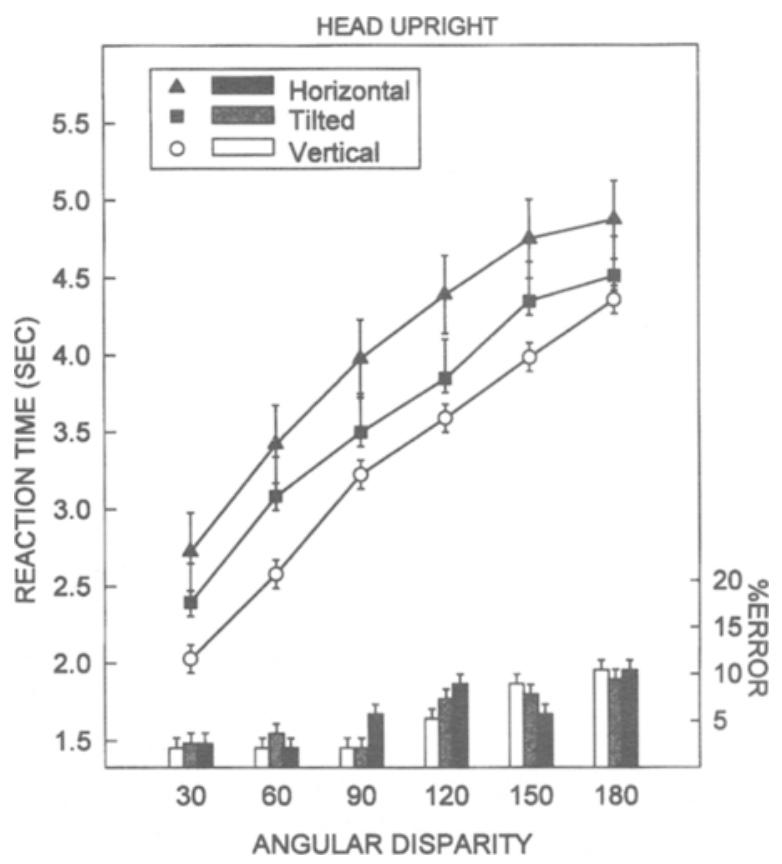

Figure 3. Reaction times and error rates on correct same trials for the head-upright condition in Experiment 2. The error bars represent the $95 \%$ confidence intervals (Loftus \& Masson, 1994).

for tilted or horizontal stimuli for 14 of the 16 observers. When observers' heads were tilted, however, intercepts were lower for vertical stimuli than for tilted stimuli for 5 observers, were approximately equal to the intercepts for tilted stimuli for 3 observers, and were higher than the intercepts for tilted stimuli for 8 observers. Further, when observers' heads were tilted, the horizontal stimuli produced the highest intercepts for all but 2 observers. The difference in intercepts between the head-upright and headtilted conditions as a function of distance from the vertical showed up as a reliable quadratic component in the interaction between head tilt and distance from the vertical $[F(1,15)=4.62, p<.05]$. This interaction was also reliable for the overall mean RTs on same trials $[F(1,15)=$ $4.85, p<.05]$.

For different trials, in this one instance, when observers' heads were upright, there was no difference between the intercepts for the vertical and tilted stimuli. Since observers can detect a difference between the stimuli at any point in the task, intercept effects (or their absence) on different trials are not critical, but it is interesting to note that the difference in intercepts between vertical and horizontal stimuli and between the tilted and horizontal stimuli were reliable for both the head-upright and head-tilted conditions.

There were no effects of vertical distance or head position on the slopes of the angular disparity functions per se (all $F \mathrm{~s}<1$ ). The slopes for vertical, tilted, and horizontal stimuli were $15.5,14.0$, and $14.4 \mathrm{msec} / \mathrm{deg}$ in the headupright condition, and $17.9,18.5$, and $15.6 \mathrm{msec} / \mathrm{deg}$ in the head-tilted condition.

To examine which of the three theoretical alternatives was the most viable, we conducted hierarchical regression 


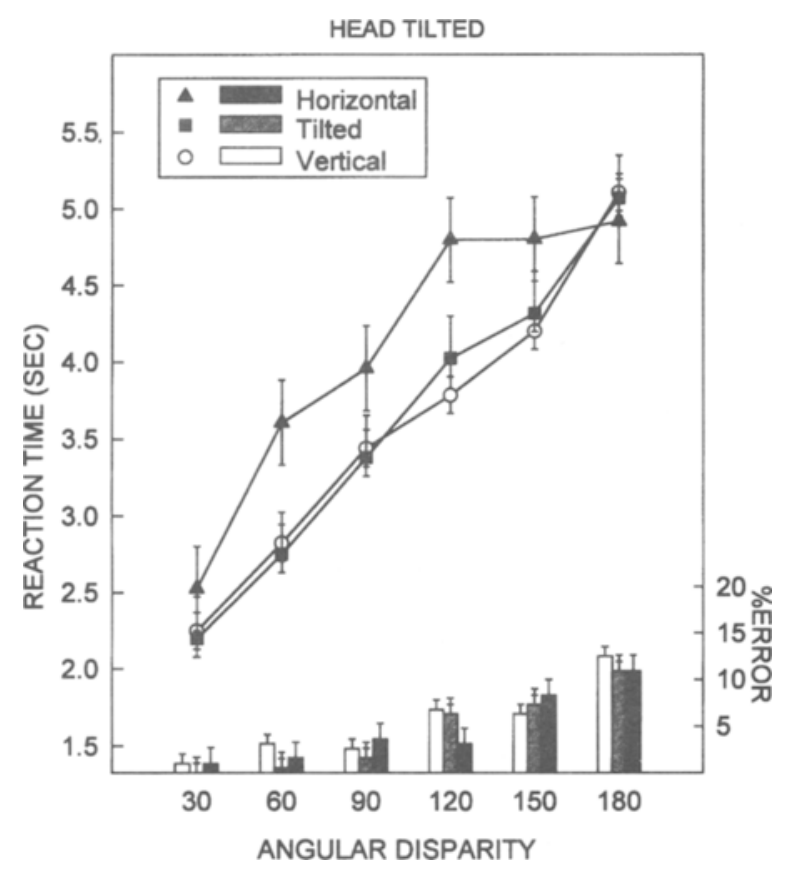

Figure 4. Reaction times and error rates on correct same trials for the head-tilted condition in Experiment 2. The error bars represent the $95 \%$ confidence intervals (Loftus \& Masson, 1994).

analyses separately for the head-upright and head-tilted conditions. For the upright condition, after the $84.1 \%$ $[F(1,16)=84.78, p<.001]$ of the variance attributable to angular disparity had been partialed out, an additional (unique) $13.3 \%$ of the remaining variance $[F(1,15)=75.19$, $p<.001]$ was accounted for by vertical distance. The parameter estimates for the head-upright condition were similar to those obtained in the previous experiment: $14.6 \mathrm{msec} /$ deg for angular disparity $\left(\beta_{1 j}\right)$ and $8.9 \mathrm{msec} / \mathrm{deg}$ for vertical distance $\left(\beta_{2 k}\right)$.

For the head-tilted condition, there were three ways to assign the levels of the vertical distance factor. In the first scheme, we assumed that observers referenced the stimuli solely to the environmental upright. In this scheme, the values for distance from the vertical were the same as in the head-upright condition: $0^{\circ}, 45^{\circ}$, and $90^{\circ}$, respectively, for the vertical, tilted, and horizontal stimuli. In the second scheme, we assumed that observers referenced the stimuli with respect to themselves; in this case, the tilted stimuli have a value of $0^{\circ}$, and the horizontal and vertical stimuli each have values of $45^{\circ}$. Finally, we could assume that observers used both frames of reference, in which case both the vertical and tilted stimuli have values of $0^{\circ}$, and the horizontal stimuli have a value of $45^{\circ}$.

In all three schemes, of course, the angular disparity factor entered the regression first and accounted for the same amount of variance $[89.1 \% ; F(1,16)=130.99, p<$ $.001]$; the parameter estimate for the angular disparity factor $\left(\beta_{1 j}\right)$ was $17.3 \mathrm{msec} / \mathrm{deg}$. The scheme that accounted for the most remaining variance was the one that assumed that observers used both the environmental and retinal frames; in this analysis, distance from the vertical accounted for
$6.2 \%$ of the remaining variance $[F(1,15)=19.47, p<$ $.001]$, and the parameter estimate $\left(\beta_{2 k}\right)$ was $11.0 \mathrm{msec} /$ deg. The scheme in which the conditions were weighted according to the environmental upright did not fare badly; distance from the vertical accounted for $4.7 \%$ of the remaining variance in this case $[F(1,15)=11.49, p<.01]$ and the parameter estimate was $5.6 \mathrm{msec} / \mathrm{deg}$. However, for the scheme in which the tilted stimuli were weighted at $0^{\circ}$ and the horizontal and vertical stimuli weighted equally at $45^{\circ}$, distance from the vertical in fact accounted for no further variance. Thus, the assumption that observers used either the environmental frame of reference alone, or more likely, some combination of the environmental and retinal frames, provided the best fit to the data.

\section{GENERAL DISCUSSION}

Observers encoded vertically oriented 3-D stimuli equally fast whether or not their heads were tilted. Similarly, observers were equally slow to encode horizontally oriented stimuli in both head-position conditions, even though horizontal stimuli are $45^{\circ}$ closer to the retinal upright when the head is tilted than when the head is upright. Data from these two conditions alone would lead us to conclude that the frame of reference used to encode the stimuli was environmentally based. However, when the stimuli were tilted, observers were faster to encode them when their heads were also tilted than when their heads were upright. Consequently, there was a clear influence of retinal coordinates in this condition.

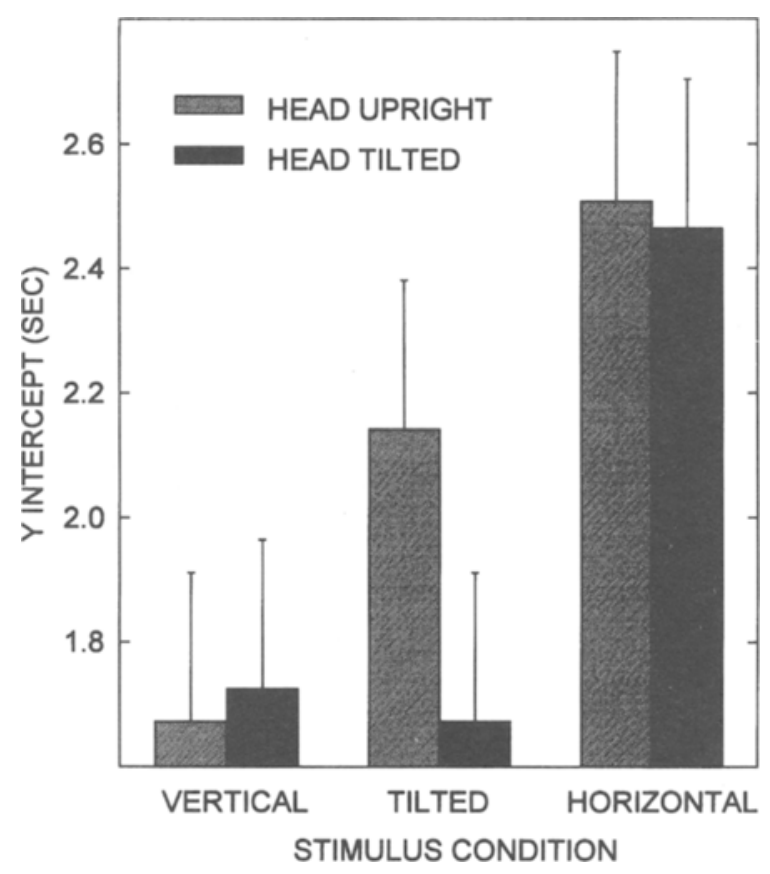

Figure 5. Intercepts derived from reaction times on correct same trials for the head-upright and head-tilted conditions in Experiment 2. The error bars represent the $95 \%$ confidence intervals (Loftus \& Masson, 1994). In this graph, the error bars are based on the mean square error term for the interaction from the analysis of variance. 
Table 3

Intercepts (in Milliseconds) and Planned Comparison Results for Same and Different Trials for Experiment 2

\begin{tabular}{|c|c|c|c|c|}
\hline & \multicolumn{2}{|c|}{ Same } & \multicolumn{2}{|c|}{ Different } \\
\hline & $\begin{array}{c}\text { Head } \\
\text { Upright }\end{array}$ & $\begin{array}{l}\text { Head } \\
\text { Tilted }\end{array}$ & $\begin{array}{l}\text { Head } \\
\text { Upright }\end{array}$ & $\begin{array}{l}\text { Head } \\
\text { Tilted }\end{array}$ \\
\hline Vertical & 1,672 & 1,725 & 3,325 & 3,26 \\
\hline Tilte & 2,1 & & &, 164 \\
\hline Horizontal & 2,508 & 2,464 & 3,882 & 4,658 \\
\hline Horizontal-vertical & $836^{a}$ & $739^{d}$ & $557 \mathrm{~g}$ & $1,398^{j}$ \\
\hline Tilted-vertical & $470^{b}$ & $-53^{\mathrm{e}}$ & $-12^{h}$ & $-96^{k}$ \\
\hline Horizontal-tilted & $366^{\mathrm{c}}$ & $792^{\mathrm{f}}$ & $569^{i}$ & $1,494^{1}$ \\
\hline \multicolumn{5}{|c|}{$\begin{array}{l}\mathrm{a}^{\mathrm{a}} F(1,15)=17.46, p<.01 . \quad \mathrm{b} F(1,15)=9.62, p<.025 . \quad \mathrm{c} F(1,15)= \\
3.54, p<.10 . \quad \mathrm{d} F(1,15)=11.60, p<.01 . \quad \mathrm{c} F(1,15)=0.06, \text { n.s. } \\
\mathrm{f} F(1,15)=9.29, p<.025 . \quad \mathrm{g} F(1,15)=8.92, p<.025 . \quad \mathrm{h} F(1,15)= \\
0.01, \text { n.s. i } F(1,15)=5.98, p<.05 . \quad \mathrm{j} F(1,15)=7.13, p<.025 . \\
{ }^{\mathrm{k}} F(1,15)=0.50, \text { n.s. } \quad{ }^{1} F(1,15)=8.07, p<.025 .\end{array}$} \\
\hline
\end{tabular}

Because the vertical stimuli did not become more difficult to encode in the head-tilted condition but the tilted stimuli became easier, it is as if observers could take advantage of the knowledge that their heads were tilted in two ways: First, knowing that their heads were tilted (and in which direction and by what amount) allowed the observers to know the particular way in which vertical stimuli were not aligned with their retinal reference frame, so they could continue to encode vertical stimuli relatively quickly. Second, the observers could encode tilted stimuli relatively quickly because tilted stimuli were aligned with their retinal reference frame, so that the relation between the tilted stimuli and the environmental upright was given by the relation between the observer and the environmental upright. In other words, when observers' heads were tilted, tilted stimuli were congruent with their subjective reference frame, vertical stimuli were congruent with the gravitational reference frame, and horizontal stimuli were congruent with neither.

It should be noted that in all conditions across the two experiments, there were clear effects of the misorientation of the stimuli with respect to each other. That is, observers were obviously influenced in the usual way by the angular disparity between the stimuli. Further, because observers still had to proceed with the mental rotation process even when their heads were tilted, the effects of the head-tilt manipulation were not contaminated by the need to rotate the stimuli around the viewer's line of sight (as it is when stimuli are 2-D). But just as the head-tilt manipulation never influenced the slope of the response time function, the misorientation of the stimuli with respect to each other was not responsible for the intercept effects. In addition, because stimuli were always aligned with each other along their length, having to locate common parts on the two figures was not an important factor in the obtained intercept differences (see also Hall \& Friedman, 1994, Experiment 2). It also seems unlikely that the intercept differences were due to postrotation processes such as decision, because in contrast to encoding processes, it is unclear how decision processes could be systematically related to the orientation of the stimuli, nor is it clear why decision processes would be affected by head tilt in some vertical distance conditions but not others. Finally, because our previous experiments that only used a single rotation axis (Friedman \& Pilon, 1994) allow us to rule out the possibility that particular axes of rotation are responsible for the encoding time differences obtained at different distances from the upright, we can conclude that it is the necessity to rotate or refer the orientation of the figure as a whole to the environmental upright that contributes most to the obtained intercept differences.

Some researchers have argued that mental rotation is not necessary for object recognition per se, but for determining the left-right orientation of an object, or for discriminating an object from its mirror image (Corballis, 1988; Corballis \& Cullen, 1986). Although this view no longer seems entirely tenable (McMullen \& Farah, 1991; McMullen \& Jolicoeur, 1990, 1992; Tarr, 1995; Tarr \& Pinker, 1990, 1991), even if observers in our study were only referencing stimuli to the environment because the shape identification task required a mirror image discrimination, it is instructive to note that having to reference 3-D vertically oriented stimuli to the environment is done at no cost when an observer's head is tilted. This implies that the gravitational vector itself, rather than, or in addition to, visual cues, may be used in the computation. Some recent data are consistent with this supposition.

Treisman and Gormican (1988) found that when observers were upright, a search asymmetry existed for orientation information: Searching for a line tilted $18^{\circ}$ in a field of vertical distractors was done in parallel (i.e., the tilted target "pops out"), but searching for a vertical line among a field of tilted distractors was done serially. They suggested that orientation information is coded in early vision in relation to standard reference values, such as the vertical and the horizontal. More recently, Marendaz, Stivalet, Barraclough, and Walkowiac (1993) extended the task as well as Treisman and Gormican's conclusions, by showing that orientation processing in early vision integrates visual and nonvisual information: When observers are supine, so that gravitational information becomes irrelevant to the task, the search asymmetry disappears and both vertical and $18^{\circ}$ targets exhibit pop-out. Foster and Ward (1991) had previously proposed a psychophysical model of orientation processing in which the maximum response of the vertical filters was greater than that of the horizontal filters. Marendaz et al. concluded that the horizontal and vertical orientation filters proposed by Foster and Ward are not immutably specified in terms of retinal coordinates, but rather, may be tuned dynamically in response to an observer's determination of his/her own general spatial orientation. In the present context, the implication is that observers normally integrate visual information and gravitational/postural information early in processing, so that they can certainly take account of their head position in relation to the gravitational vector at little or no cost.

The present data suggest that there might be two processes of normalization underlying performance in this task: The first process aligns or refers the pair of objects to the environmental upright (if necessary), and the second brings the objects into alignment with each other. This 
is similar to what Corballis and Roldan (1975) proposed their observers did to make symmetry judgments of dot patterns: First rotate the patterns to the upright, and then make the judgment. Alternatively, the data might be accounted for by a model in which observers first rotate their own egocentric frame of reference into alignment with that of the stimuli (e.g., Robertson et al., 1987), and then rotate the stimuli into alignment with each other. It is also possible that observers used an environmental frame on some trials and a retinal frame on others, resulting in a mixture model (Robertson et al., 1987). Finally, observers might neither rotate the stimuli nor their subjective reference frames to the upright, but might change the representation of the stimulus to reflect where the top is and its position relative to the upright. The present experiments were not designed to distinguish between transforming the stimuli or their representations versus transforming the subjective reference frame, or between "pure" and "mixture" models of performance; rather, they were designed to demonstrate a clear effect of distance from the vertical on encoding times and to determine how the effect changes as a function of head tilt.

It should be noted that the findings in the present study do not address the issue of whether the frame of reference for representing familiar objects is viewer- or object-centered (e.g., Corballis, 1988; Hinton \& Parsons, 1981; Marr, 1982; Marr \& Nishihara, 1978; Tarr, 1995; Tarr \& Pinker, 1989, 1990). Because we used one shape and its mirror-image in a finite number of positions, it is possible that with more trials observers could have formed view-dependent representations and the mental rotation function would have flattened (e.g., Tarr \& Pinker, 1989; Tarr, 1995). But it is an open question whether the initial encoding time differences as a function of orientation from the upright would remain, once view-dependent representations had developed.

The findings of the present study indicate that observers are sensitive to the general orientation of unfamiliar 3-D stimuli relative to the environmental upright even when this relation is irrelevant to the mental rotation component of the shape identity judgment, and that some of the information used to set up the representation that is mentally transformed in this task is computed by using the general orientation of the observer and of the object with respect to the environmental upright. Thus, both the viewer's and the environmental frame of reference exert an influence on performance.

\section{REFERENCES}

ApPelle, S. (1972). Perception and discrimination as a function of stimulus orientation: The "oblique" effect in man and animals. Psychological Bulletin, 78, 266-278.

Asch, S. E., \& WiTKIN, H. A. (1948). Studies in space orientation: I. Perception of the upright with displaced visual fields. Journal of Experimental Psychology, 38, 325-337.

Attneave, F., \& OlSON, R. K. (1967). Discriminability of stimuli varying in physical and retinal orientation. Journal of Experimental Psychology, 74, 149-157.

Bethell-Fox, C. E., \& Shepard, R. N. (1988). Mental rotation: Effects of stimulus complexity and familiarity. Journal of Experimental Psychology: Human Perception \& Performance, 14, 12-23.
Christiansen, H. N., \& STEPhenson, M. (1983). MOVIE.BYU training text. Provo, UT: Brigham Young University Press.

COOPER, L. A., \& ShEPARD, R. N. (1973). Chronometric studies of the rotation of mental images. In W. G. Chase (Ed.), Visual information processing (pp. 75-176). New York: Academic Press.

Corballis, M. C. (1988). Recognition of disoriented shapes. Psychological Review, 95, 115-123.

Corballis, M. C., \& Cullen, S. (1986). Decisions about the axes of disoriented shapes. Memory \& Cognition, 14, 27-38.

Corballis, M. C., Nagourney, B. A., Shetzer, L. I., \& StefanATOS, G. (1978). Mental rotation under head tilt: Factors influencing the location of the subjective reference frame. Perception \& Psychophysics, 24, 263-273.

Corballis, M. C., \& Roldan, C. E. (1975). Detection of symmetry as a function of angular orientation. Journal of Experimental Psychology: Human Perception \& Performance, 1, 221-230.

Corballis, M. C., Zbrodoff, J., \& Rolddan, C. E. (1976). What's up in mental rotation? Perception \& Psychophysics, 19, 525-530.

DiLorenzo, J. R., \& Rock, I. (1982). The rod-and-frame effect as a function of the righting of the frame. Journal of Experimental Psychology: Human Perception \& Performance, 8, 536-546.

EDELMAN, S., \& BüLtHOFF, H. H. (1992). Orientation dependence in the recognition of familiar and novel views of three-dimensional objects. Vision Research, 32, 2385-2400.

EGAN, D. E. (1978). Characterizing spatial ability: Different mental processes reflected in accuracy and latency scores. Murray Hill, NJ: Bell Laboratories.

FINLEY, G. (1985). A high-speed plotter for vision research. Vision Research, 25, 1993-1997.

FosTER, D. H., \& WARD, P. A. (1991). Asymmetries in oriented-line detection indicate two orthogonal filters in early vision. Proceedings of the Royal Society of London: Series $B, \mathbf{2 4 3}, 75-81$.

Friedman, A., \& Pilon, D. J. (1994). Effects of distance between objects and distance from the vertical axis on shape identity judgments. Memory \& Cognition, 22, 552-564.

Friedman, A., Pilon, D. P., \& Gabrys, G. G. (1988). Cognitive coordinate systems for mental rotation. Paper presented at the Banff Annual Symposium in Cognitive Science, Banff, AB [Abstract published in Canadian Psychology, 29, 383].

Hall, D. L., \& FriEdman, A. (1994). Shape discriminations of threedimensional objects depend on the number and location of bends. Perception \& Psychophysics, 56, 288-300.

HeYwood, S., \& CHURCHER, J. (1980). Structure of the visual array and saccadic latency: Implications for oculomotor control. Quarterly Journal of Experimental Psychology, 32, 335-341.

Hinton, G. E., \& Parsons, L. M. (1981). Frames of reference and mental imagery. In J. Long \& A. D. Baddeley (Eds.), Attention and performance $I X$ (pp. 261-277). Hillsdale, NJ: Erlbaum.

HUMPHREY, G. K., \& JolicoeUR, P. (1988). Visual object identification: Some effects of image foreshortening and monocular depth cues. In Z. W. Pylyshyn (Ed.), Computational processes in human vision: An interdisciplinary perspective (pp. 430-444). Norwood, NJ: Ablex.

JolicoEUr, P. (1985). The time to name disoriented natural objects. Memory \& Cognition, 13, 289-303.

JoLICOEUR, P. (1988). Mental rotation and the identification of disoriented objects. Canadian Journal of Psychology, 42, 461-478.

Jolicoeur, P., Regehr, S., Smith, B. J. P., \& Smith, G. N. (1985). Mental rotation of representations of two-dimensional and threedimensional objects. Canadian Journal of Psychology, 39, 100-129.

Just, M. A., \& Carpenter, P. A. (1976). Eye fixations and cognitive processes. Cognitive Psychology, 8, 441-480.

Just, M. A., \& CARPENTER, P. A. (1985). Cognitive coordinate systems: Accounts of mental rotation and individual differences in spatial ability. Psychological Review, 92, 137-172.

LASAGA, M. I., \& GARNER, W. R. (1983). Effect of line orientation on various information-processing tasks. Journal of Experimental Psychology: Human Perception \& Performance, 9, 215-225.

LofTus, G. R., \& Masson, M. E. J. (1994). Using confidence intervals in within-subject designs. Psychonomic Bulletin \& Review, 1, 476-490.

Marendaz, C., Stivalet, P., Barraclough, L., \& Walkowiac, P. 
(1993). Effect of gravitational cues on visual search for orientation. Journal of Experimental Psychology: Human Perception \& Performance, 19, 1266-1277.

MARR, D. (1982). Vision. San Francisco: W. H. Freeman.

MARR, D., \& Nishihara, H. K. (1978). Representation and recognition of the spatial organization of three-dimensional shapes. Proceedings of the Royal Society of London: Series B, 200, 269-294.

MCMullen, P. A., \& Farah, M. J. (1991). Viewer-centered and objectcentered representations in the recognition of naturalistic line drawings. Psychological Science, 2, 275-277.

MCMullen, P. A., \& JolicoeuR, P. (1990). The spatial frame of reference in object naming and discrimination of left-right reflections. Memory \& Cognition, 18, 99-115.

MCMullen, P. A., \& Jolicoeur, P. (1992). Reference frame and effects of orientation on finding the tops of rotated objects. Journal of Experimental Psychology: Human Perception \& Performance, 18, 807-820.

METZLER, J., \& ShEPARD, R. N. (1974). Transformational studies of the internal representation of three-dimensional objects. In R. L. Solso (Ed.), Theories of cognitive psychology: The Loyola Symposium (pp. 147-201). Potomac, MD: Erlbaum.

Palmer, S. E., \& Hemenway, K. (1978). Orientation and symmetry: Effects of multiple, rotational, and near symmetries. Journal of Experimental Psychology: Human Perception \& Performance, 4, 691-702.

PARSONS, L. M. (1987). Visual discrimination of abstract mirrorreflected three-dimensional objects at many orientations. Perception \& Psychophysics, 42, 49-59.

Pilon, D. J. P., \& Friedman, A. (1995). Visual search for contour and orientation information from complete and vertex-only three-dimensional line drawings. Manuscript submitted for publication.

Robertson, L. C., Palmer, S. E., \& GomeZ, L. M. (1987). Reference frames in mental rotation. Journal of Experimental Psychology: Learning, Memory, \& Cognition, 13, 368-379.

Rock, I. (1974). Orientation and form. New York: Academic Press. Rock, I., \& HeImer, W. (1957). The effect of retinal and phenomenal orientation on the perception of form. American Journal of Psychology, 70, 493-511.

Rock, I., \& LEAMAN, R. (1963). An experimental analysis of visual symmetry. Acta Psychologica, 21, 171-183.

SHEPARD, R. N., \& METZLER, J. (1971, February 19). Mental rotation of three-dimensional objects. Science, 171, 701-703.

SHIFFrar, M. M., \& SHEPARD, R. N. (1991). Comparison of cube rotations around axes inclined relative to the environment or to the cube. Journal of Experimental Psychology: Human Perception \& Performance, 17, 44-54.

STERNBERG, S. (1969). Memory scanning: Mental processes revealed by reaction time experiments. American Scientist, 57, 421-457.

TARR, M. J. (1995). Rotating objects to recognize them: A case study on the role of viewpoint dependency in the recognition of three-dimensional objects. Psychonomic Bulletin \& Review, 2, 55-82.

TARR, M. J., \& PINKER, S. (1989). Mental rotation and orientation dependence in shape recognition. Cognitive Psychology, 21, 233-282.

TARR, M. J., \& PINKER, S. (1990). When does human object recognition use a viewer-centered reference frame? Psychological Science, 1, 253256.

TARR, M. J., \& Pinker, S. (1991). Orientation-dependent mechanisms in shape recognition: Further issues. Psychological Science, 2, 207-209.

Taumer, R., Lemb, M., \& Namislo, M. (1976). Characteristics of human saccadic eye movements in different directions. Albrect von Graefes Archiv für Klinische und Experimentelle Ophthalmologie, 200 , 163-174.

Treisman, A., \& Gormican, S. (1988). Feature analysis in early vision: Evidence from search asymmetries. Psychological Review, 95, 15-48.

WILSON, V. J., \& JONES, G. M. (1979). Mammalian vestibular physiology. New York: Plenum.

YARBus, A. L. (1967). Eye movements and vision. New York: Plenum.

Yuille, J. C., \& Steiger, J. H. (1982). Nonholistic processing in mental rotation: Some suggestive evidence. Perception \& Psychophysics, 31, 201-209.

\section{APPENDIX}

Reaction Times (RT, in Milliseconds) and Errors (E) for Different Trials in Experiments 1 and 2 as a Function of Angular Disparity, Distance From the Vertical, and Head Tilt

\begin{tabular}{|c|c|c|c|c|c|c|c|c|c|c|c|c|}
\hline & \multicolumn{12}{|c|}{ Angular Disparity } \\
\hline & \multicolumn{2}{|c|}{$30^{\circ}$} & \multicolumn{2}{|c|}{$60^{\circ}$} & \multicolumn{2}{|c|}{$90^{\circ}$} & \multicolumn{2}{|c|}{$120^{\circ}$} & \multicolumn{2}{|c|}{$150^{\circ}$} & \multicolumn{2}{|c|}{$180^{\circ}$} \\
\hline & RT & E & $\mathrm{RT}$ & E & RT & $\mathrm{E}$ & RT & E & RT & $\mathrm{E}$ & RT & $\mathrm{E}$ \\
\hline \multicolumn{13}{|c|}{ Experiment 1} \\
\hline Vertical & 4,289 & 7.6 & 4,070 & 4.9 & 4,095 & 6.3 & 4,348 & 9.3 & 4,373 & 10.4 & 4,728 & 9.7 \\
\hline Tilted & 4,246 & 5.6 & 4,408 & 3.5 & 4,451 & 4.2 & 4,822 & 5.6 & 5,010 & 8.3 & 4,865 & 15.3 \\
\hline Horizontal & 5,734 & 5.6 & 5,467 & 2.3 & 5,619 & 4.2 & 5,280 & 6.2 & 5,106 & 5.6 & 5,422 & 7.6 \\
\hline \multicolumn{13}{|c|}{ Experiment 2} \\
\hline \multicolumn{13}{|l|}{ Head Up } \\
\hline Vertical & 3,510 & 0.5 & 3,393 & 0.0 & 3,898 & 0.5 & 3,765 & 0.5 & 3,842 & 2.6 & 4,129 & 1.0 \\
\hline Tilted & 3,349 & 1.6 & 3,751 & 1.0 & 4,043 & 1.0 & 4,020 & 1.0 & 3,951 & 2.6 & 4,467 & 2.6 \\
\hline Horizontal & 4,030 & 3.6 & 4,101 & 1.0 & 4,459 & 1.0 & 4,324 & 2.6 & 4,633 & 4.7 & 4,733 & 2.1 \\
\hline \multicolumn{13}{|l|}{ Head Tilted } \\
\hline Vertical & 3,354 & 0.5 & 3,453 & 0.0 & 4,063 & 1.0 & 4,253 & 1.6 & 4,009 & 0.5 & 4,260 & 1.0 \\
\hline Tilted & 3,437 & 1.6 & 3,199 & 0.5 & 3,815 & 0.5 & 4,189 & 2.1 & 4,223 & 0.5 & 4,060 & 1.6 \\
\hline Horizontal & 4,513 & 1.0 & 4,441 & 2.6 & 4,624 & 2.1 & 4,915 & 1.6 & 4,523 & 2.1 & 4,142 & 3.6 \\
\hline
\end{tabular}

(Manuscript received January 12, 1995;

revision accepted for publication June 2, 1995.) 